\title{
ANALYSIS OF MALIGNITY RATES OF PERCUTANEOUS BIOPSY IN LYMPH NODES OF BREAST CANCER PATIENTS
}

Marina Diogenes Teixeira, Maria Isabela Bloise Alves Caldas Sawada, Anna Lya Menezes Martins, Andre Mattar ${ }^{1}$, Luiz Henrique Gebrim¹

${ }^{1}$ Hospital Pérola Byington - São Paulo (SP), Brazil.

Introduction: The evaluation of regional lymph nodes in patients with breast cancer is one of the main predictive and prognostic factors for treatment. The methods of percutaneous evaluation of suspicious lymph nodes frequently used are fine-needle aspiration (FNA) cytology and core-needle biopsy (CNB). According to the international literature, CNB and FNA are considered diagnostic methods with high specificity ( $98 \%$ vs. $99 \%$ ). H, the FNA may present up to $21 \%$ of inconclusive results by insufficient material. Although $\mathrm{CNB}$ is well established as a percutaneous method for diagnostic evaluation of suspected breast lesions, the literature is scarce on the use of this technique for the evaluation of suspicious lymph nodes in breast cancer patients. Objectives: Analyze the positivity of FNA and CNB performed in suspicious lymph nodes for breast cancer metastasis according to the anatomical location of biopsies and the type of needle used, verifying which technique was preferred. Methods: A retrospective study was conducted by evaluating the database of patients treated in the non-palpable lesions sector of a public hospital in São Paulo. Women submitted to ultrasound-guided percutaneous biopsy of lymph nodes from May 2015 to November 2019 were included in the study. The data were analyzed using IBM-SPSS version 27 and Microsoft EXCEL version 2010. Results: A total of 499 biopsies were performed, and the mean age of the women was 54.2 years ( $\mathrm{SD} \pm 11.9$ ) in the CNB group and 53.4 years ( $\mathrm{SD} \pm 11.8$ ) in the FNA group ( $\mathrm{p}=0.619)$. According to the anatomical location, 385 were axillary (77.2\%), 62 supraclavicular (12.4\%), 48 cervical (9.6\%) and 4 infraclavicular (0.8\%). Regarding the type of needle, 393 were CNBs (78.8\%) and 106 were FNA (21.2\%). When analyzing the results of the FNA, 38 (35.8\%) did not present enough material, 31 (29.2\%) were positive, $32(30.2 \%)$ were negative and $5(4.8 \%)$ showed atypical cells. Among the 393 CNBs performed, 255 (64.9\%) were positive, 132 (33.6\%) were negative, 1 (0.3\%) showed atypical cells and $5(1.3 \%)$ had no representative material. No complications were reported after the procedures. Conclusions: CNB was the preferred diagnostic technique in our service, being considered a feasible procedure to evaluate lymph nodes in different sites and with low rates of inconclusive results due to insufficient material. In the future, studies evaluating indirect costs may confirm the feasibility of CNB in patients with suspicious lymph nodes in terms of obtaining greater agility and resolutive conducts in the public healthcare system. 